\section{In search of better therapies to prevent device-induced thrombosis}

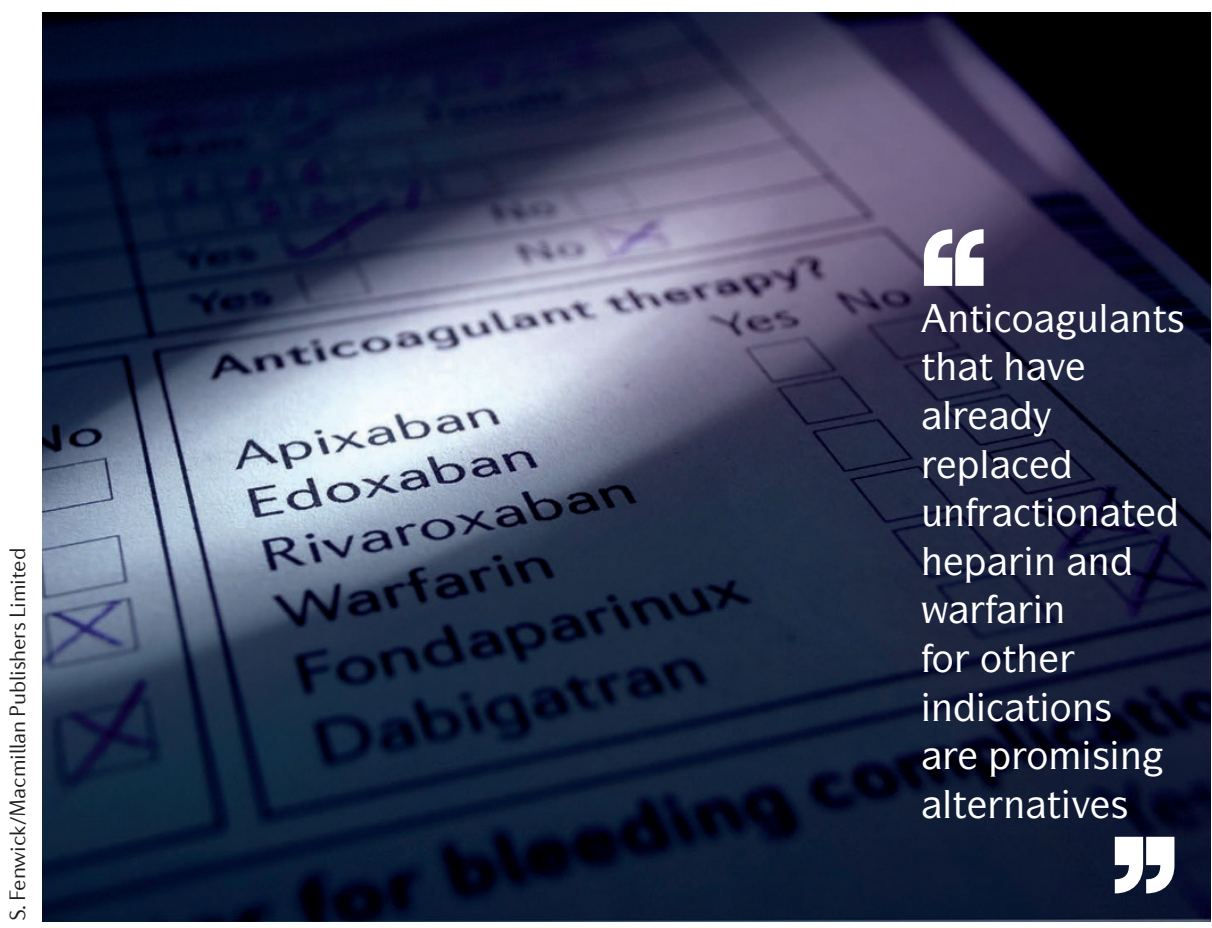

Blood-contacting medical devices such as mechanical heart valves and catheters are thrombogenic. Thrombosis can be triggered, for example, via the tissue factor released from damaged tissues during surgery or by the activation of the contact pathway of coagulation by exposure of the blood to the artificial prosthetic surface. Consequently, patients exposed to these devices usually require anticoagulation therapy to prevent device failure and thromboembolic complications.

Therapy with unfractionated heparin or with a vitamin $\mathrm{K}$ antagonist such as warfarin is the standard therapy to prevent medicaldevice-induced thrombosis. However, unfractionated heparin and warfarin have several limitations, including the need for routine coagulation monitoring, as well as multiple food and drug interactions that necessitate food, alcohol, and drug restrictions. Therefore, effective anticoagulants with a more predictable dose-response are needed for the prevention of medical-device thrombosis.

Anticoagulants that have already replaced unfractionated heparin and warfarin for other indications are promising alternatives to prevent thrombosis on prosthetic surfaces. Fondaparinux, a synthetic pentasaccharide that inhibits factor $\mathrm{Xa}$, was shown to be an effective and safe alternative to unfractionated heparin for the prevention and treatment of venous thrombosis and for the treatment of patients with acute coronary syndrome (MILESTONE 8). However, findings from the OASIS- 5 and OASIS- 6 trials showed that the risk of catheter-induced thrombosis was higher with the use of fondaparinux than with the use of unfractionated heparin or low-molecular weight heparin (LMWH) in patients who underwent percutaneous coronary intervention.

Oral direct anticoagulants - such as dabigatran (a thrombin inhibitor) and apixaban, edoxaban, and rivaroxaban (factor Xa inhibitors) - have demonstrated efficacy and safety in the prevention of stroke in patients with atrial fibrillation and the prevention and treatment of venous thromboembolism (MILESTONE 10). Initial studies in animal models demonstrated the efficacy of direct inhibition of thrombin with dabigatran in preventing valve thrombosis. However, the RE-ALIGN trial designed to assess the use of dabigatran in patients with mechanical heart valves had to be terminated early because therapy with dabigatran was associated with increased rates of bleeding complications compared with use of adjusted-dose warfarin. In addition, even at the high doses used in the trial (300 mg twice daily), dabigatran was less effective than warfarin at preventing thromboembolic complications. Warfarin might be more effective than dabigatran because warfarin suppresses coagulation at many levels (via inhibition of the activation of tissue-factor-induced coagulation and contact-pathway-induced coagulation, as well as the synthesis of factor $\mathrm{X}$ and thrombin in the common pathway). By contrast, dabigatran inhibits only thrombin. If the resulting thrombin concentrations are high, it can overwhelm the local levels of dabigatran.

Use of direct factor Xa inhibitors might be a better option for inhibiting thrombosis, because each molecule of factor Xa induces the generation of 1,000 molecules of thrombin; therefore, inhibition of factor Xa might attenuate thrombin generation to similar levels as warfarin. Indeed, preclinical findings with apixaban and rivaroxaban are promising. Oral administration of rivaroxaban was superior to subcutaneous administration of LMWH in preventing prosthetic heart valve thrombosis in a porcine model. Similarly, oral or intravenous administration of apixaban was as effective as administration of adjusted-dose warfarin in preventing valve thrombosis in a porcine model of heterotopic aortic valve. Nevertheless, further studies in animal models and human clinical trials are needed before apixaban or rivaroxaban can be considered a safer alternative to current therapies in humans.

Other promising approaches to prevent device-induced thrombosis have focused on rendering artificial surfaces less thrombogenic. Examples of these approaches include the modification of the surface to resist adsorption of blood proteins and cells (for example, by modulating electrostatic and hydrophobic interactions between the adsorbed protein and the artificial surface or by coating the devices with trypsin inhibitor) and the inhibition of thrombin generation and fibrin formation, for example, by grafting antithrombotic agents onto the biomaterial surface.

Irene Fernández-Ruiz, Senior Editor, Nature Reviews Cardiology

\footnotetext{
ORIGINAL ARTICLES Mehta, S. R. et al. Efficacy and safety of fondaparinux versus enoxaparin in patients with acute coronary syndromes undergoing percutaneous coronary intervention: results from the OASIS-5 trial.J. Am. Coll. Cardiol. 50, 1742-1751 (2007)|Eikelboom, S. J.et al. Dabigatran versus warfarin in patients with mechanical heart valves. N. Engl. J. Med. 369, 1206-1214 (2013)

FURTHER READING Jaffer, I. H. et al. Medical device-induced thrombosis: what causes it and how can we prevent it? J. Thromb. Haemost. 13 (Suppl. 1), S72-S81 (2015) | Jaffer, I. H. et al. Anticoagulation for mechanical heart valves: will oral factor Xa inhibitors be effective? Arterioscler. Thromb. Vasc. Biol. 37, 743-745 (2017)
} 